\title{
Optimal Distribution Strategy for Movie Product
}

\author{
Jingpei Ma*, Wenli Li \\ School of Economics and Management, Dalian University of Technology, Dalian, China
}

Email address:

majingpei@mail.dlut.edu.cn (Jingpei Ma),wlli@dlut.edu.cn (Wenli Li)

${ }^{*}$ Corresponding author

\section{To cite this article:}

Jingpei Ma, Wenli Li. Optimal Distribution Strategy for Movie Product. International Journal of Economics, Finance and Management Sciences. Vol. 8, No. 1, 2020, pp. 31-38. doi: 10.11648/j.ijefm.20200801.14

Received: November 22, 2019; Accepted: January 9, 2020; Published: February 3, 2020

\begin{abstract}
Motion picture companies are increasingly providing movies online. We develop a model to examine optimal distribution strategies for the movie studio. The studio can release the movie through the traditional channel (i.e. theatre) or online channel and has to decide the distribution strategy. When the studio is vertically integrated with the exhibitor, our results indicate that in the presence of a relatively low cost of using a traditional channel, the dual-channel strategy exists and generates the highest profit. However, when the cost is relatively high, the online channel strategy becomes the best strategy. When the studio is not vertically integrated with the exhibitor, when the studio's commission share is low, the studio will adopt the dual-channel strategy if the traditional channel cost is low and the quality difference is large. If not, the studio will adopt the online channel strategy. When the studio's commission share is moderate, the studio will adopt the dual-channel strategy if the traditional channel cost is low and the quality difference is large, the studio will adopt the dual-channel strategy if the traditional channel cost is low and the quality difference is moderate, otherwise, the studio will adopt the online channel strategy. When the studio's commission share is large, the studio has more motivation to adopt traditional strategy.
\end{abstract}

Keywords: Movie Studio, Exhibitor, Movie Distribution Channel, Dual Channel

\section{Introduction}

Movies are typically shown in theatres. With the advent of the Internet, a dramatic change has taken place in how movie studios manage film distribution. It is increasingly popular for movie studios to provide their movie product to customers directly through the Internet, a channel strategy referred to as an online channel strategy. The movie studio can employ a dual-channel strategy to offer movies through the two channels simultaneously or sequentially, allowing the consumers to decide on which channel to participate.

The supply chain of movie products is composed as follows: content providers (movie producers), movie studios and channel vendors (exhibitors), which correspond to the production, distribution, and screening of movie products. At the first stage, film producers need to develop new (or adapted existing) scripts, shoot directors and final products. After the movie is produced, it enters the second stage-distribution, the movie studios face a variety of marketing decisions, including advertising budget, advertising methods, and channel selection. In the third stage, with the continuous advancement of digital technology, exhibitors can display movie products through traditional theatres, and can also be screened through various media such as the internet, television, mobile phones, and DVDs.

In this paper, for the convenience of research, first of all, in terms of channels, it means that movie products are only shown through traditional channels and online channels. The traditional channel screening refers to exhibitor submitting the film to the theatre, and the theatre is responsible for the movie screening; the online channel screening means that the film issuer publishes the movie resource on the network, and the consumer can watch the movie by paying for the click. this paper mainly focusing on the channel strategy of the movie studio: including which channel the movie will be launched--only in the traditional channel, only in the online channel or dual channel.

The objective of this research is to help the industry managers understand the consequences of the changes in the distribution agreements. Specifically, we aim to address the following research questions, namely, which channel strategy is optimal for distributing movie products when the studio is vertically integrated with the exhibitor, and which channel 
strategy is optimal for distributing movie products when the studio is non-integrated with the exhibitor?

The rest of the paper is as follows. Section 2 reviews the relevant literature. Section 3 explores the studio's optimal distribution channel strategy. Section 4 conduct several numerical studies. Section 5 concludes the paper.

\section{Literature Review}

Our research is related to several streams of prior research. Many studies focus on characterizing the conditions under which a firm should implement both physical and digital channels [1-4]. Several scholars explored the pricing strategies under the setting of dual channel [5-9]. In line with their research, we found that the optimal pricing policies for the movie studio.

Eliashberg et al. (2006) provide an extensive review of research related to the motion picture industry [10]. In their discussion of the distribution stage, they pose several questions about the substitutability of DVDs for theatrical consumption and how consumers make trade-offs between the two product forms. We investigate these topics at the consumer level to study various distribution strategies.

There is an extensive repertoire of studies on various aspects of the pricing and distribution strategies of digital goods. Demirkan and Cheng (2008) study an application services supply chain consisting of one application service provider (ASP) and one application infrastructure provider (AIP) [11]. The ASP buys computer capacity from the AIP and then sells the valued-added software services to the market. They examine the supply chain's performance under different coordination strategies involving risk and information sharing between the ASP and the AIP and find an effective decentralized mechanism to achieve the goal of maximizing the overall supply chain performance. Extending the work of Demirkan and Cheng (2008), Demirkan et al. (2010) analyze the coordination mechanisms in a SaaS supply chain by explicitly taking into account the congestion cost of computing [12]. One interesting finding of their analysis is that the congestion cost in accessing the SaaS service and computing capacity costs affect the overall surplus more severely when the supply chain partners follow coordinated strategies than when they do not. However, little of this stream of research addresses the issues of movie product in the supply chain setting. The notable exceptions are Calzada and Valletti (2012) [13]. They show that versioning can be optimal for information goods with zero marginal costs. They further establish that a monopolist, or a centralized channel, will often price and simultaneously release both versions. Our research differs from Calzada and Valletti (2012) because we consider the aggregate cost of using a traditional channel.

In the movie industry, theatre exhibitors do not want too-short video windows, to avoid some consumers waiting for the video version. However, producers and video distributors might prefer a quicker video release, as this moves their video revenues ahead and increases the benefits of publicity. Although some papers such as Corts (2001),
Mortimer (2007), and Gil (2009) take into account the vertical separation between the producer and the channels, to our knowledge, this is the first work that analyzes the movie studio's distribution strategy under both vertical separation and vertically integrated cases [14-16].

\section{The Model}

In this section, we model the movie studio's optimal channel strategy problem. We consider a movie studio that offers two versions of a movie product, a theater-version denotes as $t$ and an online-version denotes as e. the theater-version is showed through theater in the traditional channel and the online-version is shown directly in the online channel, respectively.

Let $q_{t}$ and $q_{e}$ denote the quality of theater-version and online-version, respectively. Since in the movie industry, the quality of the theatrical version is allegedly higher than the one of the online version, we assume that $q_{e}<q_{t}$. For example, the film Avatar would be associated with a higher value in the theatres because of its special effects and 3-D features.

There is a continuum of consumers who are heterogeneous in their preferences over quality. Each consumer's preference type $\theta$ is uniformly distributed in the interval $[0,1]$. We assume that the movie can be consumed under the traditional channel (in theater-version) for a given price $p_{t}>0$, and consumed under the online channel (online-version) for $p_{e}>0$. Let $c$ denotes the aggregate cost of using the traditional channel, which includes match cost for watching a particular movie at a given runtime, traffic cost of the time spent to go to the theatre, waiting cost in the theatre before the movie starting,..., and so on. To exclude trivial cases, we assume that the aggregate cost cannot be higher than the quality of the theatrical version, i.e., $c<q_{t}$.

Then, given the movie quality level $q_{t}$ and $q_{e}$ and the prices of the movie $p_{t}$ and $p_{e}$, consumers choose from three options: (i) go to the theatre to watch the movie, (ii) watch the movie online, or (iii) forgo watch completely. More specifically, the utility of a consumer watch the movie in the theatre with valuation $\theta$ is:

$$
U_{t}=\theta q_{t}-p_{t}-c
$$

The utility of a consumer watching the movie under the online channel is

$$
U_{e}=\theta q_{e}-p_{e}
$$

At times it will be convenient to refer to $x=q_{e} / q_{t}<1$, which denotes the "quality ratio" of watching the movie through the two different channels. For simplicity, we let $q_{t}=1$. Without loss of any generality, we normalize the market size to one and assume that each consumer demands at most one unit of the movie product, either from the traditional channel or the online channel.

The movie studio may or may not be vertically integrated with the theatre. We examine both cases as follows. 


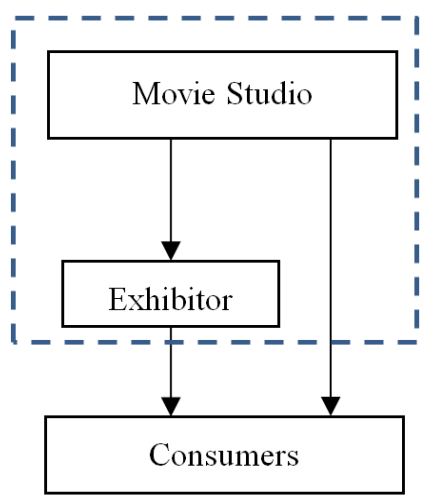

(a) Integration case

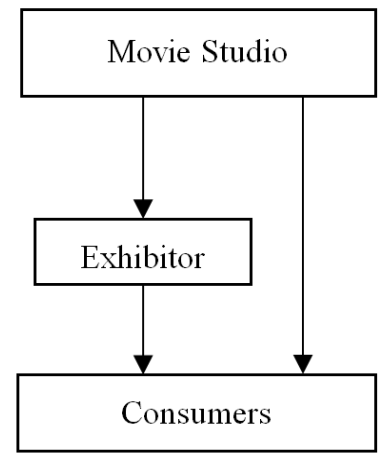

(b) Non-integration case
Figure 1. Channel structure under two cases

\subsection{A Vertically Integrated Studio}

We first analyze the case where the studio adopts the theatre channel and the studio is vertically integrated with the exhibitor (see Figure 1(a)). It is customary to divide the movie industry into three vertically related sectors: production, distribution, and exhibition. Production and distribution are often performed by the same studios, which we call studios in our model. "Exhibitors," by contrast, run theatres and screen movies to attract audiences.

The marginal consumer indifferent between watching the movie in the theatre and doing without is derived by setting the utility $U_{t}$ in Eq. (1) to zero. Then, demand equals $1-p_{t}-c$.

The studio's profit function is then described by Eq. (3).

$$
\pi_{B}^{\mathrm{A} 1}=p_{t}\left(1-p_{t}-c\right)
$$

The studio's optimal price and profit are $p_{t}{ }^{* \mathrm{~A} 1}=\frac{1-c}{2}$ and $\pi_{B}{ }^{* \mathrm{~A} 1}=\frac{(1-c)^{2}}{4}$.

We next study the case when the studio adopts the online channel strategy. The marginal consumer is derived by setting $U_{e}$ in Eq. (2) to zero. Then the demand equals $1-\frac{p_{e}}{x}$.

The studio's profit function under the online channel is then described by Eq. (4).

$$
\pi_{B}^{\mathrm{A} 2}=p_{e}\left(1-\frac{p_{e}}{x}\right)
$$

The studio's optimal price and profit are $p_{e}{ }^{* \mathrm{~A} 2}=\frac{x}{2}$ and $\pi_{B}{ }^{* \mathrm{~A} 2}=\frac{x}{4}$.

When the studio adopts the dual-channel strategy, the customers have the freedom to choose the theatre or the online channel. The marginal consumer type who is indifferent between participating in the online channel and not in either channel is $\theta_{0}=\frac{p_{e}}{x}$. The marginal consumer type who is indifferent between participating in the online channel and the theater equals $\theta_{1}=\frac{p_{t}+p_{e}-c}{1-x}$. For the dual-channel to exist, $\theta_{0} \leq \theta_{1}$ is required; otherwise, the problem reduces to a theatre channel problem if $\theta_{1}>\theta_{0}$. The $\theta_{0} \leq \theta_{1}$ requirement leads to $\frac{p_{e}}{x} \leq p_{t}+c$ and implies that it becomes a constraint in the studio's dual-channel optimization problem.

We thus have $Q_{t}=1-\frac{p_{t}+p_{e}-c}{1-x}, Q_{e}=\frac{x\left(c+p_{t}\right)-p_{e}}{(1-x) x}$. The studio's profit function is

$$
\pi_{B}^{\mathrm{A} 3}=p_{t}\left(1-\frac{p_{t}+p_{e}-c}{1-x}\right)+p_{e}\left[\frac{x\left(c+p_{t}\right)-p_{e}}{(1-x) x}\right]
$$

The studio solves the following problem.

$$
\max _{p_{t}, p_{e}} \pi_{B}^{\mathrm{A} 3}=p_{t}\left(1-\frac{p_{t}+p_{e}-c}{1-x}\right)+p_{e}\left[\frac{x\left(c+p_{t}\right)-p_{e}}{(1-x) x}\right]
$$

Subject to:

$$
\begin{gathered}
\theta_{0} \leq \theta_{1} \\
\theta_{1} \leq 1
\end{gathered}
$$

Where $\theta_{0}$ and $\theta_{1}$ are the marginal consumers defined above. Solving the firm's optimization problem, we obtain Lemma 1.

Lemma 1: There are two sets of solutions for the studio's optimal prices and profit depending on the relationship between $x$ and $c$.

When $c \leq 1-x$, the solutions are $p_{t}{ }^{* \mathrm{~A} 3}=\frac{1-c}{2}, p_{e}{ }^{* \mathrm{~A} 3}=\frac{x}{2}$, and $\pi_{B}{ }^{* \mathrm{~A} 3}=\frac{1+c^{2}-x+2 c(x-1)}{4-4 x}$.

When $c>1-x$, however, the dual-channel effectively reduces to an online channel. The studio's optimal price and profit become $p_{e}{ }^{* \mathrm{~A} 3}=\frac{x}{2}$ and $\pi_{B}{ }^{* \mathrm{~A} 3}=\frac{x}{4}$ as in the pure online channel case.

The managerial implications of the condition $c>1-x$ in Lemma 1 are as follows. Recall from Eq. (1) that $c$ is the aggregate cost of using the traditional channel, while $x$ is the "quality ratio" of watching the movie through the two different channels. That is, a high cost and a small difference will lead to no customers participating in the theatre channel. The dual strategy will in effect reduce the online channel strategy.

Comparing the profit achieved under the dual-channel strategy with those of the theatre and online channel strategies, we obtain Proposition 1:

Proposition 1 (Studio's Optimal Sequencing Strategy, Vertically Integrated): when $c \leq 1-x$, the dual-channel strategy exists, and it generates a higher profit than the theater and online channel strategies. If $c>1-x$, the dual-channel reduces to an online channel strategy, and the online channel dominates the theater channel.

We next study the studio's optimal profit of each channel strategy where there exists a separate exhibitor in the channel.

\subsection{With the Existence of an Exhibitor}

In this scenario, there exists an exhibitor to run theatres and screen movies to attract audiences (see Figure 1(b)).

In the traditional channel, the studio and the exhibitor are in an agency arrangement. The exhibitor sets the retail price $\left(p_{t}\right)$ and pays the studio a fraction $\alpha \in[0,1]$ of the movie revenues. In the online channel, the studio offers movie directly and sets the retail price $\left(p_{e}\right)$.

We first investigate the case where the studio adopts the dual-channel strategy. From the setting, the profits for the studio $\left(\pi_{\mathrm{st}}^{\mathrm{A} 3}\right)$ and the exhibitor $\left(\pi_{\mathrm{ex}}^{\mathrm{A} 3}\right)$ can be written as: 


$$
\pi_{\mathrm{st}}^{\mathrm{A} 3}=\alpha p_{t}\left(1-\frac{p_{t}+p_{e}-c}{1-x}\right)+p_{e}\left[\frac{x\left(c+p_{t}\right)-p_{e}}{(1-x) x}\right]
$$

Subject to:

$$
\begin{gathered}
\theta_{0} \leq \theta_{1} \\
\theta_{1} \leq 1
\end{gathered}
$$

and

$$
\pi_{\mathrm{ex}}^{\mathrm{A} 3}=(1-\alpha)\left(1-\frac{p_{t}+p_{e}-c}{1-x}\right) p_{t}
$$

The firms set the retail prices - the exhibitor sets $p_{t}$ and the studio, $p_{e}$ - to maximize their profits. The second-order conditions, $d^{2} \pi_{e x}{ }^{A 3} / d p_{t}{ }^{2}=-\frac{2(1-\alpha)}{1-c}<0$ and $d^{2} \pi_{\mathrm{st}}{ }^{\mathrm{A} 3} /$ $\mathrm{dp}_{e}{ }^{2}=-\frac{2}{1-x}-\frac{2}{x}<0$, are also satisfied. Simultaneously solving the first-order conditions $d \pi_{e x}{ }^{A 3} / d p_{t}=0$ and $d \pi_{s t}{ }^{A 3} / d p_{e}=0$, we obtain the equilibrium prices and profit for the studio under the dual channel. This lead to the following lemma.

Lemma 2: 1) When $c \leq 2(1-x) /(2-x), p_{t}{ }^{* \mathrm{~A} 3}=$ $[2(1-c-x)+c x] /(4-x-x \alpha), \quad p_{e}{ }^{* \mathrm{~A} 3}=x[c(1-\alpha)+$ $(1-x)(1+\alpha)] /(4-x-x \alpha)$, and $\pi_{\mathrm{st}}{ }^{* \mathrm{~A} 3}=[(1+c-$ $x)^{2} x+(1-x)(4-4 x+c(4 c-(x-4) x-8)) \alpha-(1-$ c) $\left.(x-1)(c+x-1) x \alpha^{2}\right] /(1-x)(x+x \alpha-4)^{2}$. 2) When $c>2(1-x) /(2-x)$, there is the only demand through online channel, and the dual channel reduces to the online channel.

When the studio adopts the traditional channel, the exhibitor decides the retail price $\left(p_{t}\right)$. The exhibitor's profit function is given by $\pi_{\mathrm{ex}}^{\mathrm{A} 1}=(1-\alpha)\left(1-p_{t}-c\right) p_{t}$. The optimal retail price is derived as $p_{t}{ }^{* \mathrm{~A} 1}=\frac{1-c}{2}$. The studio's profit function is given by $\pi_{\mathrm{st}}{ }^{\mathrm{A} 1}=\alpha\left(1-p_{t}-c\right) p_{t}$. Plugging in $p_{t}{ }^{* \mathrm{~A} 1}=\frac{1-c}{2}$, we obtain that $\pi_{\mathrm{st}}{ }^{* \mathrm{~A} 1}=\frac{(1-c)^{2} \alpha}{4}$. When the studio adopts the online channel, the results remain the same as in Section 3.1. Thus, $p_{e}^{* \mathrm{~A} 2}=\frac{x}{2}, \pi^{* \mathrm{~A} 2}=\frac{x}{4}$.

Comparing the optimal profits under the three channel strategies, we obtain the following proposition:

Proposition 2(Studio's Optimal Distribution Strategy, Not Vertically Integrated): The studio's optimal channel strategy is the following.

(1) If $0<\alpha<0.376$

When $0<c<c^{\text {", }}$, the studio choose dual-channel strategy; When $c^{\prime \prime}<c \leq \frac{2(1-x)}{2-x}$, the studio chooses online channel strategy.

(2) If $0.376<\alpha<1$

When $0<c<c^{\prime}$, the studio choose traditional channel strategy; When $c^{\prime}<c<c^{\prime \prime}$, the studio choose dual-channel strategy;

When $c^{\prime \prime}<c \leq \frac{2(1-x)}{2-x}$, the studio choose online channel strategy.

Where $\quad c^{\prime \prime}=\frac{(1-x)\left(8 \alpha+x^{2} \alpha(1+\alpha)-2 x(1+\alpha)^{2}\right)-x \sqrt{(1-x)(x+x \alpha-4)^{2}}}{2\left(4 \alpha+x^{2} \alpha^{2}-x\left(-1+4 \alpha+\alpha^{2}\right)\right)}$ and $c^{\prime}=\frac{2 \sqrt{x \alpha(1-x)(x+x \alpha-4)^{2}}-(1-x)(4-x \alpha)\left(1-\alpha^{2}\right)}{4+\left(8-9 x+x^{2}\right) \alpha+2\left(2-3 x+x^{2}\right) \alpha^{2}-(1-x) x \alpha^{3}}$.

A closer look at Proposition 2 shows that, unlike the integration case, the optimal channel strategy is also affected by the commission share ratio with the existence of an exhibitor. When the studio shares a lower proportion of commissions in the traditional channels, its channel strategy is similar to the integration case; otherwise, when the commission share is higher than 0.376 , the time cost of the consumer in the traditional channel is lower, and the difference in the quality between the two versions is in the $\left[\frac{4(1-\alpha)^{2}}{4+(1-\alpha)^{2} \alpha}, \alpha\right]$ interval, the studio will choose to adopt the traditional channel strategy. Furthermore, the higher the commission ratio, the larger the range above.

Therefore, Proposition 2 provides some management implications for movie studios and exhibitors. For movie studios, they could adjust their channel strategies flexibly according to different commission rates. For the exhibitors, first of all, they could combine its screening quality advantages and provide consumers with a better viewing environment to improve the quality of theater-version; secondly, by optimizing site selection and provide online ticket sales, they could reduce the time cost of consumers and enhance their channel competitiveness. Finally, the exhibitors could adjust their pricing strategy according to different commission ratios.

\section{Numerical Analysis}

In this section, to verify the aforementioned analysis, we conduct several numerical studies. And we set the parameters variation range as shown in Table 1.

Table 1. The intervals of parameters.

\begin{tabular}{ll}
\hline Parameter & Variation Range \\
\hline$c$ & 0.4 \\
$x$ & 0.6 \\
$\alpha$ & {$[0.15,0.4,0.5]$} \\
\hline
\end{tabular}

First, we analyze how the parameters influence the channel strategy of the studio under the integration case. Figure 2 depicts how the parameters $c$ and $x$ affect the studio's channel strategy under the integration case. Specifically, let $c=0.6$, Figure 2 (a) analyzes the changes in profits of studio under traditional channel strategies, online channel strategies, and dual-channel strategies as $x$ changes. As can be seen from the figure $2(a), 1)$ Under the dual-channel strategy and the online channel strategy, as the $x$ increases, the difference in the quality of two versions is reduced, and the profit of the studio increases; 2) when $x<0.4$, the studio achieves the most profit under the dual-channel strategy; when $x \geq 0.4$, the two profit functions completely coincided because the studio changes from the dual-channel strategy to the online channel strategy. Let $x=0.4$, Figure 2 (a) analyzes the changes in profits of the studio under different strategies as $c$ changes. As can be seen from Figure $2(\mathrm{~b}), 1$ ) when $\mathrm{c}<0.4$, the profit of the studio increases under the dual-channel strategy and the traditional channel strategy as the $c$ decreases; 2) when $c \geq 0.4$, the two profit functions completely coincided. 

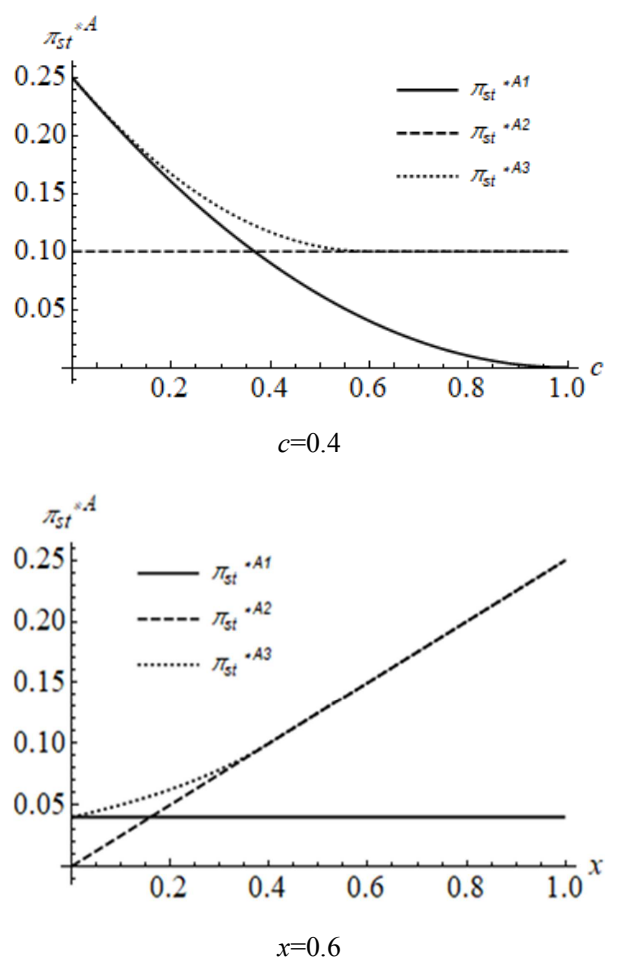

Figure 2. The studio's profit for fixed and randomly varying parameters.

Figure 3 depicts the optimal channel strategy for the studio in two-dimensional space ( $\boldsymbol{c}$ and $\boldsymbol{x}$ ) under the integration case. As consumers spend more costs on traditional channels and the difference in the quality of the two versions becomes smaller, the studio gradually shifts from dual-channel strategy to online channel strategy.

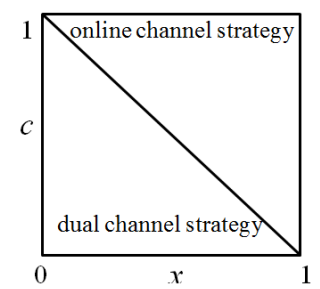

Figure 3. The studio's equilibrium channel strategy under the integration case as a function of $c$ and $x$.

Next, we analyze how the parameters influence the channel strategy of the studio under the non-integration case.

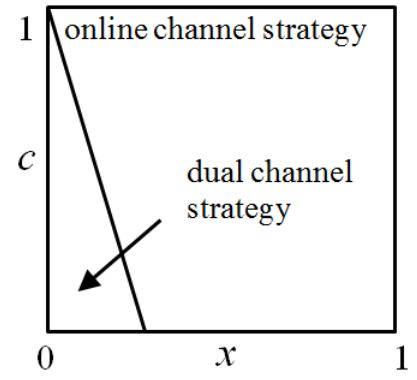

(a) $\alpha=0.15$

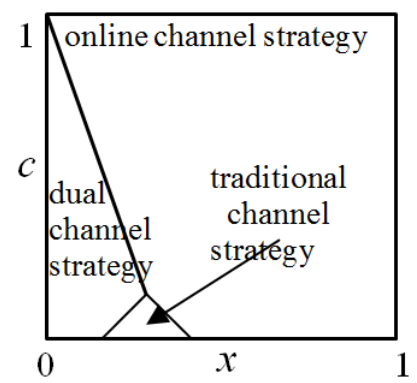

(b) $\alpha=0.4$

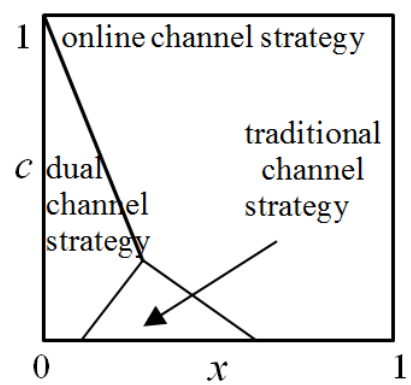

(c) $\alpha=0.5$

Figure 4. The studio's equilibrium channel strategy under the non-integration case as a function of $c$ and $x$.

Figure 4 depicts the optimal channel strategy for the studio in two-dimensional space ( $c$ and $x$ ) under non-integration case when $\alpha$ changes. Specifically, let $\alpha=0.15$, From Figure 4(a) we can see that, when the studio has a smaller percentage of commissions in traditional channels $(\alpha=$ 0.15 ), the studio gradually shifts from dual-channel strategy to online channel strategy as consumers spend more cost on traditional channels and the difference in the quality of the two versions becomes smaller. From Figure 4(b) and 4(c) we can see that, when the studio has a large proportion of commissions in the traditional channel $(\alpha=0.4, \alpha=0.5)$, it may transfer the online channel strategy to the traditional channel strategy.

\section{Conclusion}

We have presented a model of movie distribution and consumption across two channels that provide insights on how studios should make optimal distribution decisions. Our results indicate that in the presence of relatively low cost of using traditional channel, the dual-channel strategy exists and generates the highest profit. Otherwise, when the cost is relatively high, the online channel strategy becomes the best strategy when the studio is integrated with the exhibitor. The traditional channel strategy can be the best strategy when the studio is not integrated with the exhibitor.

\section{Acknowledgements}

Jingpei Ma gratefully acknowledges the support of National Natural Science Foundation of China (Grant \#: 71431002, 71731003). 


\section{Appendix}

Proof of Lemma 1:

The studio's decision problem under the dual channel is

$$
\max _{p_{t}, p_{e}} \pi^{\mathrm{A} 3}=p_{t}\left(1-\frac{p_{t}+p_{e}-c}{1-x}\right)+p_{e}\left[\frac{x\left(c+p_{t}\right)-p_{e}}{(1-x) x}\right]
$$

Subject to:

$$
1-\frac{p_{t}+p_{e}-c}{1-x} \geq 0, \frac{x\left(c+p_{t}\right)-p_{e}}{(1-x) x} \geq 0
$$

The Lagrange equation can be written as:

$$
L=p_{t}\left(1-\frac{p_{t}+p_{e}-c}{1-x}\right)+p_{e}\left[\frac{x\left(c+p_{t}\right)-p_{e}}{(1-x) x}\right]+\left[\frac{x\left(c+p_{t}\right)-p_{e}}{(1-x) x}\right] \lambda_{1}+\left(1-\frac{p_{t}+p_{e}-c}{1-x}\right) \lambda_{2} .
$$

There are four combinations of Lagrange multipliers to be considered:

Combination 1: $\lambda_{1}=0, \lambda_{2}=0$

By $\frac{\partial L}{\partial p_{t}}=0$ and $\frac{\partial L}{\partial p_{e}}=0$, we obtain $p_{t}{ }^{*}=\frac{1-c}{2}$ and $p_{e}{ }^{*}=\frac{x}{2}$. Put $p_{t}{ }^{*}$ and $p_{e}{ }^{*}$ into $\frac{x\left(c+p_{t}\right)-p_{e}}{(1-x) x} \geq 0, \frac{c}{2-2 x} \geq 0$ is always satisfied. Put $p_{t}{ }^{*}$ and $p_{e}{ }^{*}$ into $1-\frac{p_{t}+p_{e}-c}{1-x} \geq 0$, we obtain the condition of $c \leq 1-x$.

Combination 2: $\lambda_{1}=0, \lambda_{2}>0$

In this case, $\lambda_{2}>0$ lead to $1-\frac{p_{t}+p_{e}-c}{1-x}=0$ and thus there is no demand from the traditional channel.

By $\frac{\partial L}{\partial p_{t}}=0, \frac{\partial L}{\partial p_{e}}=0$ and $1-\frac{p_{t}+p_{e}-c}{1-x}=0$, we obtain that $p_{t}{ }^{*}=1-c-\frac{x}{2}, p_{e}{ }^{*}=\frac{x}{2}$ and $\lambda_{2}{ }^{*}=c-1+x$. Put $p_{t}{ }^{*}$ and $p_{e}{ }^{*}$ into $\frac{x\left(c+p_{t}\right)-p_{e}}{(1-x) x} \geq 0, \frac{1}{2} \geq 0$ is always satisfied. 。

The condition $\lambda_{2}>0$ lead to $c>1-x$.

Combination 3: $\lambda_{1}>0, \lambda_{2}=0$

$\lambda_{1}>0$ indicates that there is no demand from the online channel. By $\frac{\partial L}{\partial p_{t}}=0, \frac{\partial L}{\partial p_{e}}=0$ and $\frac{x\left(c+p_{t}\right)-p_{e}}{(1-x) x}=0$, we obtain that $p_{t}{ }^{*}=\frac{1-c}{2}, p_{e}{ }^{*}=\frac{x(1+c)}{2}$ and $\lambda_{1}{ }^{*}=-c x$. For $\lambda_{1}{ }^{*}$ is negative which is contradicting to $\lambda_{1}>0$, this case is infeasible.

Combination 4: $\lambda_{1}>0, \lambda_{2}>0$

Under this case, there is no demand from either channel and thus this case is not optimum.

The second-order condition is shown as follows. The Hessian matrix can be written as

$$
H=\left(\begin{array}{cc}
\frac{\partial^{2} \pi_{s}}{\partial p_{t}{ }^{2}} & \frac{\partial^{2} \pi_{s}}{\partial p_{t} \partial p_{e}} \\
\frac{\partial^{2} \pi_{s}}{\partial p_{e} \partial p_{t}} & \frac{\partial^{2} \pi_{s}}{\partial p_{e}{ }^{2}}
\end{array}\right)=\left(\begin{array}{cc}
\frac{-2}{1-x} & \frac{2}{1-x} \\
\frac{2}{1-x} & \frac{-2}{x(1-x)}
\end{array}\right)
$$

Since $\frac{\partial^{2} \pi_{s}}{\partial p_{t}{ }^{2}}=-\frac{2}{1-x} \leq 0$ and $-\frac{2}{1-x} \cdot \frac{-2}{x(1-x)}-\frac{2}{1-x} \cdot \frac{2}{1-x} \geq 0$, we obtain $-\frac{2}{1-x} \leq 0$ and $-\frac{2}{1-x} \cdot \frac{-2}{x(1-x)}-\frac{2}{1-x} \cdot \frac{2}{1-x} \geq 0$ are always satisfied, so the optimal solutions obtained above are for a maximization problem.

Proof of Proposition 1:

When $c \leq 1-x, \pi^{* \mathrm{~A} 3}=\left[1+c^{2}-x+2 c(x-1)\right] /(4-4 x), \pi^{* \mathrm{~A} 1}=\frac{(1-c)^{2}}{4}$ and $\pi^{* \mathrm{~A} 2}=\frac{x}{4}$. By comparing $\pi^{* \mathrm{~A} 3}$ and $\pi^{* \mathrm{~A} 1}$, we obtain that $\pi^{* \mathrm{~A} 3}-\pi^{* \mathrm{~A} 1}=\frac{c^{2} x}{4(1-x)}>0$.

Then, by comparing $\pi^{* \mathrm{~A} 3}$ and $\pi^{* \mathrm{~A} 2}$, we obtain that

$$
\pi^{* \mathrm{~A} 3}-\pi^{* \mathrm{~A} 2}=\frac{(1-c-x)^{2}}{4(1-x)}>0
$$

hence, when $c \leq 1-x$, the dual-channel strategy always yields a higher profit than the traditional channel strategy, if the dual-channel strategy exists.

When $c>1-x$, the dual-channel reduces to an online channel. By comparing $\pi^{* \mathrm{~A} 2}$ and $\pi^{* \mathrm{~A} 1}$, we obtain that $\pi^{* \mathrm{~A} 2}-$ $\pi^{* \mathrm{~A} 1}=\frac{x-(1-c)^{2}}{4(1-x)}$.

Since $0<x<1,(1-c)^{2}<x$ is always satisfied. Hence, when $c>1-x$, the online channel strategy always yields the highest profit.

Proof of Proposition 2:

First, we compare $\pi_{\mathrm{st}}{ }^{* \mathrm{~B} 3}$ and $\pi_{\mathrm{st}}{ }^{* \mathrm{~B} 1}$. 
When $c \leq 2(1-x) /(2-x)$, we obtain $\pi_{\mathrm{st}}{ }^{* \mathrm{~B} 3}-\pi_{\mathrm{st}}{ }^{* \mathrm{~B} 1}=\frac{x Z(c)}{4(1-x)(4-x-x \alpha)^{2}}$, where $Z(c)=c^{2}\left[4(1+\alpha)^{2}+x^{2} \alpha(1+\alpha)^{2}-\right.$ $\left.x \alpha(3+\alpha)^{2}\right]+2 c(1-x)(4-x \alpha)\left(1-\alpha^{2}\right)+(1-x)\left[(1-\alpha)^{2}(4-x \alpha)-4 x\right] \quad$. Since $\quad 4(1+\alpha)^{2}+x^{2} \alpha(1+\alpha)^{2}-$ $x \alpha(3+\alpha)^{2}>0$ and $(1-x)(4-x \alpha)\left(1-\alpha^{2}\right)>0$ are always satisfied, hence, $Z(c)$ is convex.

When $x<\frac{4(1-\alpha)^{2}}{4+(1-\alpha)^{2} \alpha}, Z(0)=(1-x)\left[(1-\alpha)^{2}(4-x \alpha)-4 x\right]>0$ is always satisfied, hence, $\pi_{\mathrm{st}}{ }^{* \mathrm{~B} 3}>\pi_{\mathrm{st}}{ }^{* \mathrm{~B} 1}$.

When $x>\frac{4(1-\alpha)^{2}}{4+(1-\alpha)^{2} \alpha}, Z(0)<0$ is always satisfied. We consider two cases:

(1) $\frac{4}{4+\alpha}<x<1$. Since $Z\left(\frac{2(1-x)}{2-x}\right)=(1-x) x(x+x \alpha-4)^{2}(4-x(4+\alpha)) /(2-x)^{2}<0$ is always satisfied, hence, $\pi_{\mathrm{st}}{ }^{* \mathrm{~B} 1}>\pi_{\mathrm{st}}{ }^{* \mathrm{~B} 3}$.

(2) $\frac{4(1-\alpha)^{2}}{4+(1-\alpha)^{2} \alpha}<x<\frac{4}{4+\alpha}$. Since $Z\left(\frac{2(1-x)}{2-x}\right)=(1-x) x(x+x \alpha-4)^{2}(4-x(4+\alpha)) /(2-x)^{2}<0$, there is a unique point $c^{\prime} \in\left(0, \frac{2(1-x)}{2-x}\right)$ that makes $Z(c)=0$, where $c^{\prime}=\frac{2 \sqrt{x \alpha(1-x)(x+x \alpha-4)^{2}}-(1-x)(4-x \alpha)\left(1-\alpha^{2}\right)}{4+\left(8-9 x+x^{2}\right) \alpha+2\left(2-3 x+x^{2}\right) \alpha^{2}-(1-x) x \alpha^{3}}$. Hence, when $c \in\left(0, c^{\prime}\right]$, we obtain $Z(c)<0$ and $\pi_{\mathrm{st}}{ }^{* \mathrm{~B} 1}>\pi_{\mathrm{st}}{ }^{* \mathrm{~B} 3}$; when $c \in\left(c^{\prime}, \frac{2(1-x)}{2-x}\right]$, we obtain $Z(c)>0$ and $\pi_{\mathrm{st}}{ }^{* \mathrm{~B} 3}>\pi_{\mathrm{st}}{ }^{* \mathrm{~B} 1}$.

Second, we compare $\pi_{\mathrm{st}}{ }^{* \mathrm{~B} 3}$ and $\pi_{\mathrm{st}}{ }^{* \mathrm{~B} 2}$.

When $c \leq \frac{2(1-x)}{2-x}$, we have $\pi_{\mathrm{st}}{ }^{* \mathrm{~B} 3}-\pi_{\mathrm{st}}{ }^{* \mathrm{~B} 2}=\frac{-Y(c)}{4(1-x)(4-x-x \alpha)^{2}}$, Where $Y(c)=c^{2}\left(4(1-x) x \alpha^{2}-4 x-16(1-x) \alpha\right)+$ $c\left(8 x^{2}-8 x+32(1-x) \alpha-16(1-x) x \alpha+4(1-x) x^{2} \alpha-8(1-x) x \alpha^{2}+4(1-x) x^{2} \alpha^{2}\right)+12 x-16 x^{2}+5 x^{3}-x^{4}-$ $16(1-x) \alpha+16(1-x) x \alpha-8(1-x) x^{2} \alpha+2(1-x) x^{3} \alpha+4(1-x) x \alpha^{2}-4(1-x) x^{2} \alpha^{2}+(1-x) x^{3} \alpha^{2}$.

Since $4(1-x) x \alpha^{2}-4 x-16(1-x) \alpha<0$ is always satisfied, $Y(c)$ is concave.

$Y\left(\frac{2(1-x)}{2-x}\right)=\frac{(1-x) x^{3}(4-x-x \alpha)^{2}}{(x-2)^{2}}>0$ is always satisfied too, since $Y(0)=-(1-x)\left[16 \alpha+4 x^{2}(1+\alpha)^{2}-x^{3}(1+\alpha)^{2}-\right.$ $4 x(1+\alpha)(3+\alpha)$ ], let $F(x)=Y(0)$, where $F(x)$ is the increasing function of $\mathrm{x}$. By $F(0)=-16 \alpha<0$ and $F(1)=$ $(\alpha-3)^{2}>0$, we obtain that There is a unique point $x^{\prime \prime} \in(0,1)$ that makes $F(x)=0$, where

$$
\begin{aligned}
x^{\prime \prime}= & \frac{4(1+\alpha)^{2}}{3(1+\alpha)^{2}}+\frac{2(5-\alpha)(1+\alpha)^{3}}{3(1+\alpha)^{2}\left(19+3 \sqrt{3} \sqrt{(1+\alpha)^{8}(18+(-8+\alpha) \alpha)}+\alpha(69+\alpha(87+\alpha(38+\alpha(-3+(-3+\alpha) \alpha))))\right)^{1 / 3}} \\
& -\frac{2\left(19+3 \sqrt{3} \sqrt{(1+\alpha)^{8}(18+(-8+\alpha) \alpha)}+\alpha(69+\alpha(87+\alpha(38+\alpha(-3+(-3+\alpha) \alpha))))\right)^{1 / 3}}{3(1+\alpha)^{2}} .
\end{aligned}
$$

We consider two cases:

(1) $x^{\prime \prime}<x<1$. Since $F(x)=Y(0)>0$ and $Y(c)>0$ are always satisfied, we obtain $\pi_{\mathrm{st}}{ }^{* \mathrm{~B} 3}<\pi_{\mathrm{st}}{ }^{* \mathrm{~B} 2}$;

(2) $0<x<x^{\prime \prime}$. Since $F(x)=Y(0)<0$ are always satisfied, we obtain that there is an unique point $c^{\prime \prime} \in\left(0, \frac{2(1-x)}{2-x}\right)$ that makes $Y(c)=0$, where $c^{\prime \prime}=\frac{(1-x)\left(8 \alpha+x^{2} \alpha(1+\alpha)-2 x(1+\alpha)^{2}\right)-\sqrt{(1-x) x^{2}(-4+x+x \alpha)^{2}}}{2\left(4 \alpha+x^{2} \alpha^{2}-x\left(-1+4 \alpha+\alpha^{2}\right)\right)}$. When $c \in\left(0, c^{\prime \prime}\right]$, we obtain $Y(c)<0$ and $\pi_{\mathrm{st}}{ }^{* \mathrm{~A} 3}>\pi_{\mathrm{st}}{ }^{* \mathrm{~B} 2}$; when $c \in\left(c,, \frac{2(1-x)}{2-x}\right]$, we obtain $Y(c)>0$ and $\pi_{\mathrm{st}}{ }^{* \mathrm{~B} 3}<\pi_{\mathrm{st}}{ }^{* \mathrm{~B} 2}$.

Third, we compare $\pi_{\mathrm{st}}{ }^{* \mathrm{~B} 1}$ and $\pi_{\mathrm{st}}{ }^{* \mathrm{~B} 2}$.

When $c \leq \frac{2(1-x)}{2-x}$, we obtain $\pi_{\mathrm{st}}{ }^{* \mathrm{~B} 1}-\pi_{\mathrm{st}}{ }^{* \mathrm{~B} 2}=\frac{1}{4} M(c)$,

where, $M(c)=c^{2} \alpha-2 c \alpha-x+\alpha \circ M\left(\frac{2(1-x)}{2-x}\right)=-x\left(1-\frac{x \alpha}{(2-x)^{2}}\right)<0$.

We consider two cases:

(1) $x>\alpha$, we obtain that $M(0)<0$ and $M(c)<0$ are always satisfied. Hence, $\pi_{\mathrm{st}}{ }^{* \mathrm{~B} 1}<\pi_{\mathrm{st}}{ }^{* \mathrm{~B} 2}$.

(2) $x<\alpha$. We have $M(0)>0$ 。 There is a unique point $c^{*} \in\left(0, \frac{2(1-x)}{2-x}\right)$ that makes $M(c)=0$, where $c^{*}=\frac{\alpha-\sqrt{\mathrm{x} \alpha}}{\alpha}$. Hence, when $\in\left(0, c^{*}\right], M(c)>0$, and $\pi_{\mathrm{st}}{ }^{* \mathrm{~B} 1}>\pi_{\mathrm{st}}{ }^{* B 2}$; when $c \in\left(c^{*}, \frac{2(1-x)}{2-x}\right], M(c)<0$, and $\pi_{\mathrm{st}}{ }^{* B 1}<\pi_{\mathrm{st}}{ }^{* \mathrm{~B} 2}$.

Fourth, we make the whole analysis.

When $c<\frac{2(1-x)}{2-x}$,

If $0<\alpha<0.376$, we have $0<\alpha<x^{\prime \prime}<\frac{4(1-\alpha)^{2}}{4+(1-\alpha)^{2} \alpha}<\frac{4}{4+\alpha}<1$.

For $x \in(0, \alpha)$, we have $0<c^{\prime}<c^{*}<c^{\prime \prime}<\frac{2(1-x)}{2-x}$. Hence, when $0<c<c^{\prime \prime}$, dual channel is the optimal strategy; when $c>c$, online channel is the optimal strategy.

For $x \in\left(\alpha, x^{\prime \prime}\right)$, we obtain that when $0<c<c^{\prime \prime}$, dual channel is the optimal strategy; when $c>c$, online channel is the optimal strategy.

For $x \in\left(x^{\prime \prime}, 1\right)$, we obtain that online channel is the optimal strategy.

If $0.376<\alpha<0.828$, we have $0<\frac{4(1-\alpha)^{2}}{4+(1-\alpha)^{2} \alpha}<x^{\prime \prime}<\alpha<\frac{4}{4+\alpha}<1$. 
For $x \in\left(0, \frac{4(1-\alpha)^{2}}{4+(1-\alpha)^{2} \alpha}\right), 0<c^{\prime}<c^{*}<c^{\prime \prime}<\frac{2(1-x)}{2-x}$. We obtain that when $0<c<c^{\prime \prime}$, dual channel is the optimal strategy; when $c>c$ ", online channel is the optimal strategy.

For $x \in\left(\frac{4(1-\alpha)^{2}}{4+(1-\alpha)^{2} \alpha}, x^{\prime \prime}\right)$, there is an unique point $x_{1}$ that makes $0<c^{\prime}<c^{*}<c^{\prime \prime}$ when $x \in\left(\frac{4(1-\alpha)^{2}}{4+(1-\alpha)^{2} \alpha}, x_{1}\right)$ and $0<c^{\prime \prime}<$ $c^{*}<c^{\prime}<\frac{2(1-x)}{2-x}$ when $x \in\left(x_{1}, x^{\prime \prime}\right)$.

For $x \in\left(\frac{4(1-\alpha)^{2}}{4+(1-\alpha)^{2} \alpha}, x_{1}\right)$, when $0<c<c^{\prime}$, traditional channel is the optimal strategy; when $c^{\prime}<c<c$, dual channel is the optimal strategy; when $c>c$ ", online channel is the optimal strategy.

For $x \in\left(x_{1}, x^{\prime \prime}\right)$, when $0<c<c^{*}$, traditional channel is the optimal strategy; when $c>c^{*}$, online channel is the optimal strategy.

For $x \in\left(x^{\prime \prime}, \alpha\right)$, when $0<c<c^{*}$, traditional channel is the optimal strategy; whenc $>c^{*}$, online channel is the optimal strategy.

For $x \in(\alpha, 1)$, online channel is the optimal strategy.

If $0.828<\alpha<1$, we have $0<\frac{4(1-\alpha)^{2}}{4+(1-\alpha)^{2} \alpha}<x^{\prime \prime}<\frac{4}{4+\alpha}<\alpha<1$.

For $x \in\left(0, \frac{4(1-\alpha)^{2}}{4+(1-\alpha)^{2} \alpha}\right)$, we obtain that when $0<c<c$, dual channel is the optimal strategy; when $c>c$, online channel is the optimal strategy.

For $x \in\left(\frac{4(1-\alpha)^{2}}{4+(1-\alpha)^{2} \alpha}, x^{\prime \prime}\right)$, there is a unique point $x_{1}$ that makes $0<c^{\prime}<c^{*}<c^{\prime \prime}<\frac{2(1-x)}{2-x}$ when $x \in\left(\frac{4(1-\alpha)^{2}}{4+(1-\alpha)^{2} \alpha}, x_{1}\right)$.

For $x \in\left(\frac{4(1-\alpha)^{2}}{4+(1-\alpha)^{2} \alpha}, x_{1}\right)$, when $0<c<c^{\prime}$, traditional channel is the optimal strategy; when $c^{\prime}<c<c$, dual channel is the optimal strategy; when $c>c$ ", online channel is the optimal strategy;

For $x \in\left(x_{1}, x^{\prime \prime}\right)$, when $0<c<c^{*}$, traditional channel is the optimal strategy; whenc $>c^{*}$, online channel is the optimal strategy.

For $x \in\left(x^{\prime \prime}, \alpha\right)$, when $0<c<c^{*}$, traditional channel is the optimal strategy; when $c>c^{*}$, online channel is the optimal strategy.

For $x \in(\alpha, 1)$, the online channel is the optimal strategy.

\section{References}

[1] Lu Q, Shi V, Huang J. Who benefit from agency model: A strategic analysis of pricing models in distribution channels of physical books and e-books [J]. European Journal of Operational Research, 2018, 264 (3): 1074-1091.

[2] Huang W, Swaminathan J M. Introduction of a second channel: Implications for pricing and profits [J]. European Journal of Operational Research, 2009, 194 (1): 258-279.

[3] Wang C J, Chen Y J, Wu C C. Advertising competition and industry channel structure [J]. Marketing Letters, 2011, 22 (1): $79-99$

[4] Xia Y, Xiao T, Zhang G P. Distribution channel strategies for a manufacturer with complementary products $[\mathrm{J}]$. Decision Sciences, 2013, 44 (1): 39-56.

[5] Cattani K, Gilland W, Heese H S, et al. Boiling frogs: Pricing strategies for a manufacturer adding a direct channel that competes with the traditional channel $[\mathrm{J}]$. Production and Operations Management, 2006, 15 (1): 40.

[6] Hua G, Wang S, Cheng T C E. Price and lead time decisions in dual-channel supply chains $[\mathrm{J}]$. European journal of operational research, 2010, 205 (1): 113-126.

[7] Kurata H, Yao D Q, Liu J J. Pricing policies under direct vs. indirect channel competition and national vs. store brand competition [J]. European Journal of Operational Research, 2007, 180 (1): 262-281.

[8] Radhi M, Zhang G. Pricing policies for a dual-channel retailer

with cross-channel returns [J]. Computers \& Industrial Engineering, 2018, 119: 63-75.

[9] Rahmani K, Yavari M. Pricing policies for a dual-channel green supply chain under demand disruptions [J]. Computers \& Industrial Engineering, 2019, 127: 493-510.

[10] Eliashberg J, Elberse A, Leenders MA (2006) The motion picture industry: Critical issues in practice, current research, and new research directions. Marketing Sci. 25 (6): 638-661.

[11] Demirkan, H., \& Cheng, H. K. (2008). The risk and information sharing of application services supply chain. European Journal of Operational Research, 187 (3), 765-784.

[12] Demirkan, H., Cheng, H. K., \& Bandyopadhyay, B. (2010). Coordination strategies in an SaaS supply chain. Journal of Management Information Systems 26 (4), 119-143.

[13] Calzada J, Valletti TM. 2012. "Intertemporal movie distribution: Versioning when customers can buy both versions," Marketing Science (31: 4), July, pp 649-667.

[14] Corts KS. 2001. "The strategic effects of vertical market structure: Common agency and divisionalization in the U.S. motion picture industry," Journal of Economics \& Management Strategy (10: 4), October, pp 509-528.

[15] Gil R. 2009. "Revenue sharing distortions and vertical integration in the movie industry," The Journal of Law, Economics, \& Organization (25: 2), October, pp 579-610.

[16] Mortimer JH. 2007. "Price discrimination, copyright law, and technological innovation: Evidence from the introduction of DVDs," The Quarterly journal of economics (122: 3), August, pp 1307-1350. 